\title{
Effects of coadministration of low dose cannabinoid type 2 receptor agonist and morphine on vanilloid receptor 1 expression in a rat model of cancer pain
}

\author{
MINGYUE ZHANG ${ }^{1}$, MENG CHI $^{1}$, HUICHAO ZOU ${ }^{2}$, SONGYU TIAN $^{3}$, ZHAODI ZHANG $^{1}$ and GUONIAN WANG ${ }^{1,4}$ \\ Departments of ${ }^{1}$ Anesthesiology, ${ }^{2}$ Pain and ${ }^{3}$ Gynecology, Cancer Hospital of Harbin Medical University; \\ ${ }^{4}$ Pain Research Institute of Heilongjiang Academy of Medical Sciences, Harbin, Heilongjiang 150081, P.R. China
}

Received October 1, 2016; Accepted July 20, 2017

DOI: $10.3892 / \mathrm{mmr} .2017 .7479$

\begin{abstract}
Morphine is widely used as an analgesic to treat moderate to severe pain, but chronic morphine use is associated with development of tolerance and dependence, which limits its analgesic efficacy. Our previous research has showed that nonanalgetic dose of a cannabinoid type 2 (CB2) receptor agonist reduced morphine tolerance in cancer pain. A previous study showed the colocalization of CB2 and transient receptor potential vanilloid 1 (TRPV1) in human and rat dorsal root ganglia (DRG) sensory neurons. Whether coadministration of a CB2 receptor agonist and morphine could reduce TRPV1 expression in morphine-induced antinociception and tolerance in cancer pain is unclear. Therefore, we investigated the effects of coadministration of a CB2 receptor agonist AM1241 and morphine on TRPV1 expression and tolerance in cancer pain. Coadministration of AM1241 and morphine for 8 days significantly reduced morphine tolerance, as assessed by measuring paw withdrawal latency to a radiant heat stimulation, in Walker 256 tumor-bearing rats. Repeated morphine treatment for a period of 8 days induced upregulation of the TRPV1 protein expression levels in the DRG in the tumor-bearing rats, although no change in mRNA expression. Pretreatment with AM1241 reduced this morphine-induced upregulation of TRPV1 and the effect was reversed by the CB2 receptor antagonist AM630. Our findings suggest that coadministration of a CB2 receptor agonist AM1241 and morphine reduced
\end{abstract}

Correspondence to: Professor Guonian Wang, Department of Anesthesiology, Cancer Hospital of Harbin Medical University, 150 Haping Road, Nangang, Harbin, Heilongjiang 150081, P.R. China

E-mail: wangguonian609cn@aliyun.com

Abbreviations: CB2, cannabinoid type 2 receptor; DRG, dorsal root ganglion; MAPK, mitogen-activated protein kinase; TRPV1, vanilloid receptor 1; s.c., subcutaneous; i.t., intrathecal; i.p., intraperitoneal

Key words: cannabinoid type 2 receptor, morphine tolerance, cancer pain, TRPV1, dorsal root ganglion morphine tolerance possibly through regulation of TRPV1 protein expression in the DRG in cancer pain.

\section{Introduction}

Pain is one of the most common symptoms in patients with cancer (1). Morphine is one of the most commonly used drugs in the treatment of moderate and severe pain. However, the development of tolerance and dependence after chronic administration limit its use (2). Although several studies have partly elucidated the mechanisms of morphine tolerance $(3,4)$, the exact mechanisms mediating the development of tolerance remain to be completely understood. Aside from the opioid receptor-based mechanisms (3), certain studies have demonstrated that the interactions between opioid and non-opioid systems, including cannabinoids, also serve roles in the development of morphine tolerance (4).

Cannabinoid receptors are a family of $\mathrm{G}$ protein-coupled receptors and are classified into two subtypes, cannabinoid type 1 receptor $(\mathrm{CB} 1)$ and $\mathrm{CB} 2$. Modifying $\mathrm{CB} 1$ receptor activity has limited clinical potential due to adverse neurological side effects and the development of tolerance $(5,6)$. CB2 receptors are expressed by glia in the dorsal root ganglia (DRG) and spinal cord (7-9), as well as by neurons in the central $(10,11)$ and peripheral $(12)$ nervous systems. Certain studies have suggested that $\mathrm{CB} 2$ receptors are involved in the analgesic effects of repeated morphine administration in naïve and inflammatory animals $(4,13,14)$. In addition, several groups have reported that coadministration of a nonanalgesic dose of cannabinoid receptor agonist with morphine could reduce morphine antinociceptive tolerance in normal rats and animal models of neuropathic pain (15-17). The authors previously observed that intrathecal (i.t.) injection of a nonanalgetic dose of a CB2 receptor agonist potentiated the analgesic effect and alleviated tolerance to morphine in tumor-bearing rats, potentially by regulating $\mu$-opioid receptor expression in the spinal cord and DRG (18).

Vanilloid receptor 1 (TRPV1) is critical in the development of thermal and mechanical hyperalgesia in inflammatory, neuropathic and cancer pain $(19,20)$, and is involved in morphine tolerance in normal animals $(21,22)$. A previous study demonstrated the colocalization of CB2 and TRPV1 in human DRG 
sensory neurons, and observed that CB2 agonists selectively inhibited capsaicin-induced responses in human DRG sensory neurons (12). Previous studies have identified that cannabinoids can evoke antihyperalgesia and antinociception through cannabinoid receptors, primarily by inhibiting TRPV1 in peripheral sensory neurons $(23,24)$. In a cancer pain rat model, whether coadministration of a CB2 agonist and morphine attenuated TRPV1 expression, and whether this participated in morphine analgesia and tolerance, is unknown. Therefore, it was hypothesized that coadministration of a CB2 agonist AM1241 and morphine could reduce TRPV1 expression in peripheral sensory neurons of the DRG, in chronic morphine analgesia and tolerance in cancer pain. In the present study, the effects of coadministration of a CB2 agonist AM1241 and morphine on TRPV1 expression were investigated following repeated morphine administration in tumor-bearing rats. Morphine-mediated analgesia and tolerance was also tested using a radiant heat stimulation.

\section{Materials and methods}

Animals. Adult (6-weeks-old, 160-180 g, n=60) male specific-pathogen-free Wistar rats (Yisi Experimental Animal Corporation, Changchun, China) were housed in a climate-controlled room with a 12-h light/dark cycle, and were provided with food and water ad libitum. All animal experiments complied with the policies and recommendations of the International Association for the Study of Pain, and the National Institutes of Health (NIH) guidelines for the handling and use of laboratory animals. The experimental protocol of the present study was approved by the Animal Care and Use Committee of the Harbin Medical University (Harbin, China), and all possible steps were taken to avoid animal suffering at each stage of the experiment. After a 1-week habituation period, animals received Walker 256 breast carcinoma cell implantation on the plantar region of the right hind paw of each rat. The method of Walker 256 cell culture and implantation was performed as described previously (25).

Drug administration. Drug administration was performed 5 days following cell injection, when marked proliferation of tumor cells and the thermal hyperalgesia of the right hind paw could be detected $(20,25)$ Tumor-bearing rats were randomly divided into one of six groups using a random number table. For all experiments, $20 \mu 150 \%$ dimethyl sulfoxide was used as the vehicle. The rats in the 'control' and 'morphine' groups received subcutaneous (s.c.) injections of normal saline (NS; vehicle + saline group; $n=10$ ) or morphine sulfate (Northeast Pharmaceutical Group Co., Ltd., Harbin, China); $10 \mathrm{mg} / \mathrm{kg}$ /injection; volume, $1 \mathrm{ml} / \mathrm{kg}$; vehicle + morphine group; $n=10)$, respectively, twice daily (8:00 a.m. and 8:00 p.m.) for 8 days. Furthermore, separate animal groups received i.t. injections of a nonanalgesic dose of AM1241 (0.07 $\mu \mathrm{g}$; Cayman Chemical Company, Ann Arbor, MI, USA), the dose was determined as the authors described previously (18). Rats received injections via lumbar puncture at the L5-L6 intervertebral space and subcutaneous morphine (AM1241 + morphine group; $\mathrm{n}=10$ ), or $10 \mu \mathrm{g}$ CB2 antagonist AM630 (Cayman Chemical Company) with AM1241 and morphine (AM1241 + AM630 + morphine group; $\mathrm{n}=10$ ), twice daily for 8 days. In addition, the effects of AM1241 and AM630 were tested separately, the rats received i.t. injections of AM1241 (AM1241 + saline group; n=10) or AM630 (AM630 + saline group; $n=10$ ), and 30 min subsequently they received s.c. injections of NS twice daily for 8 days. AM1241 and AM630 were diluted in 50\% dimethyl sulfoxide and a volume of $20 \mu \mathrm{l}$ of each was used for the i.t. injection. AM630 was injected 30 min prior to the agonist AM1241 and the agonist was injected $30 \mathrm{~min}$ prior to morphine. On day 9 , the effect of AM1241 on analgesic tolerance to morphine was measured once all rats had received $5 \mathrm{mg} / \mathrm{kg}$ morphine (s.c.).

Behavioral testing. Morphine antinociception and tolerance was determined by measuring paw withdrawal latency to radiant heat stimulation (26). Rats were acclimated within plexiglass enclosures on a clear glass plate maintained at $26 \pm 0.5^{\circ} \mathrm{C}$. A radiant heat source (Chengdu Technology and Market Co., Ltd., Chengdu, China) was focused on the plantar surface of the hind paw. The stimulus shut off automatically when the hind paw moved. A threshold time of $30 \mathrm{sec}$ was set to prevent tissue damage. The tests were performed every morning, prior to drug administration (baseline) on the first test day and 30 min following drug administration on days 1-8. On day 9, the tests were performed 30, 60, 90 and $120 \mathrm{~min}$ following s.c. $5 \mathrm{mg} / \mathrm{kg}$ morphine injection.

Immunohistochemistry. The rats ( $\mathrm{n}=4$ for each group) received intraperitoneal (i.p.) injections of $100 \mathrm{mg} / \mathrm{kg}$ sodium pentobarbital for anesthesia following the behavioral test on day 9 . The rats were subsequently transcardially perfused with cold saline, followed by $4 \%$ paraformaldehyde in $0.1 \mathrm{M}$ PBS (pH 7.2-7.4, $4^{\circ} \mathrm{C}$ ). The $\mathrm{L} 4 \mathrm{DRG}$ ipsilateral to the tumor cell implantation site was removed and post-fixed in the above fixative for $24 \mathrm{~h}$ at $4^{\circ} \mathrm{C}$, then embedded in paraffin, and sectioned ( $4 \mu \mathrm{m}$ thickness). The sections were deparaffinized in a series of descending alcohol concentrations and the antigen was retrieved using $0.01 \mathrm{M}$ sodium citric buffer in high pressure $\left(120^{\circ} \mathrm{C}\right)$, and then incubated with $2 \mu \mathrm{g} / \mathrm{ml}$ polyclonal rabbit antibodies against TRPV1 (cat. no. PAB14852; Abnova, Taipei, Taiwan) overnight at $4^{\circ} \mathrm{C}$. The sections were incubated with biotinylated goat anti-rabbit immunoglobulin G (IgG) (cat. no. BA1003, 1:200; Boster Biological Technology, Pleasanton, CA, USA) for $20 \mathrm{~min}$ at room temperature, mounted with neutral balsam, and the morphological details were examined under an inverted microscope (Olympus Corporation, Tokyo, Japan). The positive area of the images was digitized and subjected to color threshold analysis using NIH ImageJ software version 2.1 (National Institutes of Health, Bethesda, MD, USA).

Western blotting. Western blotting was performed to detect the protein expression of TRPV1 using protein isolated from the lumbar segments of the DRG. Following the behavioral tests on day 9 , rats ( $\mathrm{n}=3$ for each group) were anesthetized using sodium pentobarbital $(60 \mathrm{mg} / \mathrm{kg})$ and were decapitated for tissue harvesting. The L3/L4/L5 DRG ipsilateral to the tumor cell injection site were dissected, frozen in liquid nitrogen and stored at $-80^{\circ} \mathrm{C}$ until further use. The DRG samples were homogenized in a volume of $200 \mu \mathrm{l}$ in ice-cold Protein Lysis Buffer (Beyotime Institute of Biotechnology, Haimen, China) containing the protease inhibitor phenylmethane sulfonyl fluoride (1 nM; Beyotime Institute of Biotechnology). The homogenate was centrifuged $\left(14,000 \times \mathrm{g}\right.$ for $20 \mathrm{~min}$ at $\left.4^{\circ} \mathrm{C}\right)$ 


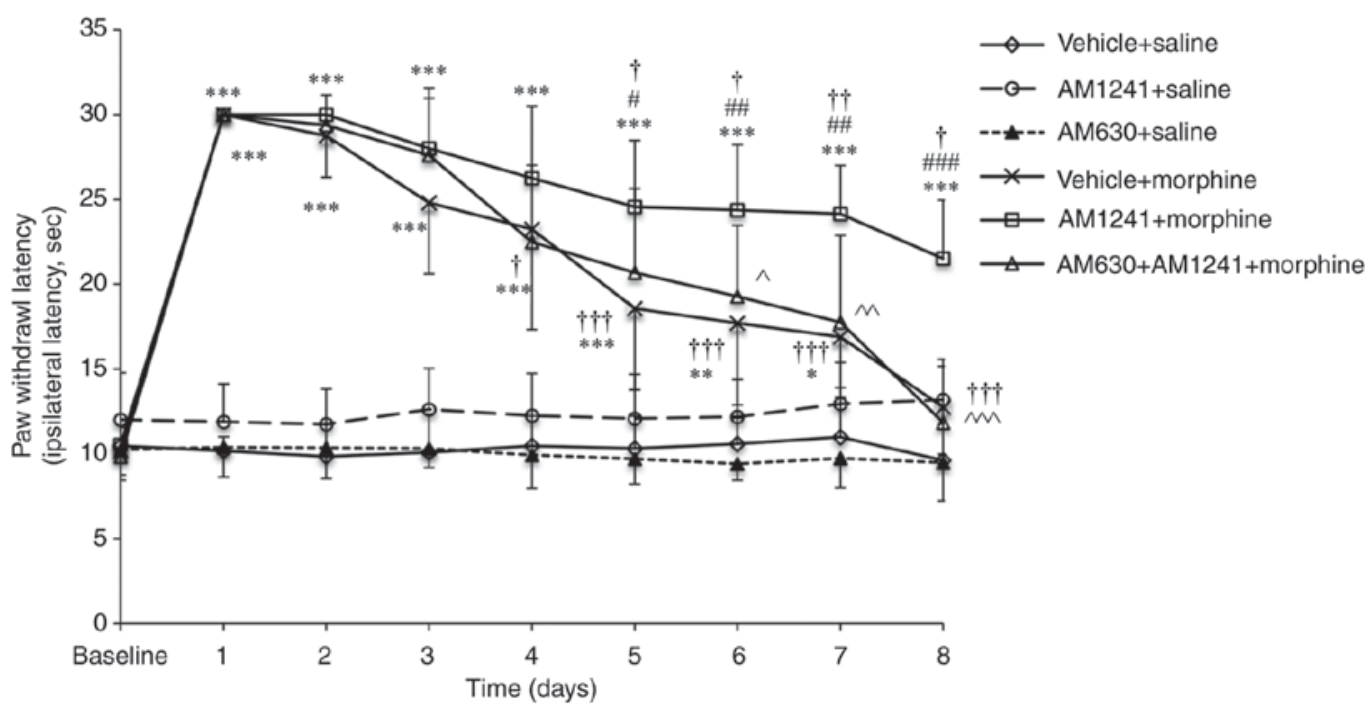

Figure 1. Effect of coadministration of AM1241 and morphine on morphine-induced antinociception. Rats received vehicle + saline, vehicle + morphine, AM1241 + saline, AM630 + saline, AM1241 + morphine or AM630 + AM1241 + morphine (n=10/group) twice daily for 8 days. The antinociceptive action of the administered drugs was evaluated 30 min following the first injection (morning) on days 1-8 of the experiment using a radiant heat stimulation. The values are presented as the mean \pm standard deviation. ${ }^{*} \mathrm{P}<0.05,{ }^{* *} \mathrm{P}<0.01,{ }^{* * * *} \mathrm{P}<0.001$ vs. the saline + vehicle group; ${ }^{\# *} \mathrm{P}<0.05,{ }^{\# \#} \mathrm{P}<0.01,{ }^{\# \# \#} \mathrm{P}<0.001$ vs. the morphine + vehicle group; ${ }^{\dagger} \mathrm{P}<0.05,{ }^{\dagger \dagger} \mathrm{P}<0.01,{ }^{\dagger \dagger} \mathrm{P}<0.001$ vs. day 1 in corresponding group; ${ }^{\wedge} \mathrm{P}<0.05,{ }^{\wedge} \mathrm{P}<0.01,{ }^{\wedge \wedge} \mathrm{P}<0.001$ AM1241 + morphine group vs. the $\mathrm{AM} 630+\mathrm{AM} 1241+$ morphine group.

and collected. Aliquots of the total protein samples (30 g; measured by BCA method) were separated by SDS-PAGE (5\% stacking gel and $12 \%$ separating gel) and then the protein was transferred onto a polyvinylidene difluoride membrane. The bands were analyzed using antibodies specific for TRPV1 (2 $\mu \mathrm{g} / \mathrm{ml}$ ) and GAPDH (cat. no. TA08, 1:500; OriGene Technologies, Inc., Beijing, China) was used as a loading control, and the membranes were incubated at $4^{\circ} \mathrm{C}$ overnight. The next day, the membranes were incubated with secondary antibodies (IgG-horseradish peroxidase, cat. nos. ZB2301 and ZB2305; OriGene Technologies, Inc.) at room temperature for $1 \mathrm{~h}$ and visualized using the ECL Plus chemiluminescence detection system (Fluorescent chemiluminescence imaging system; NatureGene Corporation, Medford, NJ, USA).

Reverse transcription-quantitative polymerase chain reaction $(R T-q P C R)$. mRNA expression of TRPV1 was analyzed by RT-qPCR using lumbar L3/L4/L5 of the DRG ipsilateral to tumor cell injection. A total of three rats from each group were anesthetized and then sacrificed immediately following behavioral tests. The L3/L4/L5 DRG were dissected and immediately frozen in liquid nitrogen and stored at $-80^{\circ} \mathrm{C}$ until further use. Total RNA was isolated using TRIzol (Invitrogen; Thermo Fisher Scientific, Inc., Waltham, MA, USA) and $2 \mu \mathrm{g}$ aliquots of the samples were used for cDNA synthesis using the Transcriptor First-Strand cDNA Synthesis kit (Roche Diagnostics, Basel, Switzerland). The synthesized cDNA was used as a template for qPCR amplification using Fast Start Universal SYBR Green Master mix (Roche Diagnostics) and the following primers: TRPV1 (Invitrogen; Thermo Fisher Scientific, Inc.) forward, 5'-TCCAAGGCACTTGCTCCA TT-3' and reverse, 5'-TGGAGGTGGCTTGCAGTTAG-3'; and GAPDH forward, 5'-AGATGGTGAAGGTCGGTGTG-3' and reverse, 5'-AACTTGCCGTGGGTAGAGTC-3'. PCR amplification was performed according to the manufacturer's protocol $\left(95^{\circ} \mathrm{C}\right.$ for $10 \mathrm{~min}$; and 40 cycles of $95^{\circ} \mathrm{C}$ for $10 \mathrm{sec}, 56^{\circ} \mathrm{C}$ for $30 \mathrm{sec}, 95^{\circ} \mathrm{C}$ for $15 \mathrm{sec}, 60^{\circ} \mathrm{C}$ for $1 \mathrm{~min}, 95^{\circ} \mathrm{C}$ for $30 \mathrm{sec}$ and $60^{\circ} \mathrm{C}$ for $15 \mathrm{sec}$ ) using an ABI 7500 fast real-time PCR system (Applied Biosystems; Thermo Fisher Scientific, Inc.).

Statistical analysis. Power analysis was based on preliminary experiments of paw withdrawal latency to radiant heat stimulation and molecular biological parameters, and yielded a sample size of $n=8$ for paw withdrawal latency to heat stimulation and $\mathrm{n}=3$ for TRPV1 protein and gene expression $(\alpha=0.05 ; 1-\beta=0.9)$ for each group. Data were analyzed using SPSS 21.0 software (version 21.0; IBM Corp., Armonk, NY, USA). The behavioral testing data were analyzed using two-way analysis of variance (ANOVA) followed by the Bonferroni post hoc test. The TRPV1 receptor protein and mRNA expression levels were analyzed by one-way ANOVA followed by post-hoc analysis using the Bonferroni test. $\mathrm{P}<0.05$ was considered to indicate a statistically significant difference. Data are presented as the mean \pm standard deviation.

\section{Results}

Effect of coadministration of AM1241 and morphine on morphine analgesia. Morphine administration produced significant antinociception between days 1 and 3 compared with the vehicle + saline group (all $\mathrm{P}<0.001)$. The effect of morphine analgesia gradually declined during chronic exposure between days 4 and 7 compared with day 1 (vehicle + morphine group: Day $4, \mathrm{P}=0.023$; days $5-8$, all $\mathrm{P}<0.001$; Fig. 1). On day 8 , the effect of chronic morphine administration on thermal withdrawal latency did not exhibit a significant difference compared with that of the vehicle + saline group (12.76 \pm 2.78 vs. $9.62 \pm 2.14 \mathrm{sec}$; $\mathrm{P}=0.431$; Fig. 1$)$, indicating that the rats developed tolerance to the analgesic effects of morphine. Rats pretreated with the nonanalgesic 


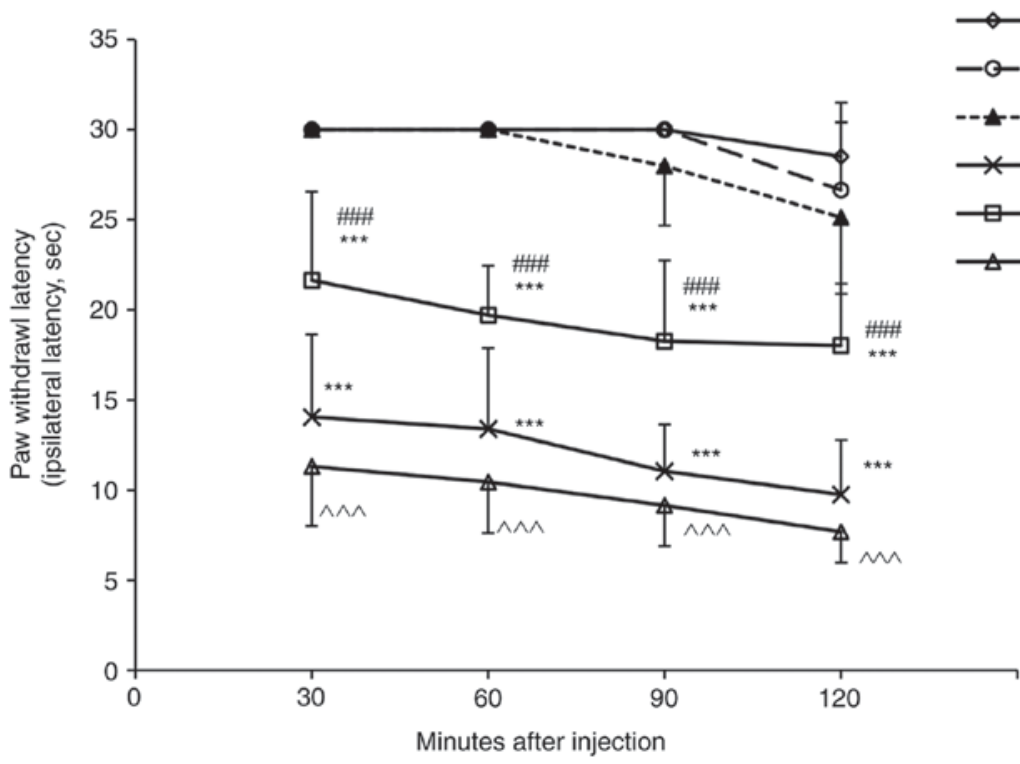

Figure 2. Effect of coadministration of AM1241 and morphine on morphine-induced analgesic tolerance. Rats received vehicle + saline, vehicle + morphine, AM1241 + saline, AM630 + saline, AM1241 + morphine or AM630 + AM1241 + morphine (n=10/group) twice daily for 8 days. On day 9 , the rats received $5 \mathrm{mg} / \mathrm{kg}$ morphine (subcutaneous) to detect the effects of AM1241 on morphine tolerance, and the paw withdrawal latency was detected 30, 60, 90 and 120 min following morphine injections. The values are presented as the mean \pm standard deviation. ${ }^{* * *} \mathrm{P}<0.001$ vs. the saline + vehicle group; ${ }^{\# \# t} \mathrm{P}<0.001$ vs. the morphine + vehicle group; ${ }^{\wedge \wedge} \mathrm{P}<0.001 \mathrm{AM} 1241+$ morphine group vs. the AM630 + AM1241 + morphine group.

dose of AM1241 (AM1241 + morphine group) exhibited no statistically significant difference compared with morphine alone (vehicle + morphine group) on morphine-induced analgesia between days 1 and 4 . However, on day 5 , the effect of morphine was significantly reduced in the vehicle + morphine group compared with that of the AM1241 + morphine group (18.58 \pm 4.80 vs. $24.56 \pm 3.92 \mathrm{sec} ; \mathrm{P}=0.024$; Fig. 1$)$. Although the paw withdrawal latency reduced in the following days in AM1241 + morphine group, the effects of morphine analgesia remained significantly increased in the AM1241 + morphine group compared with the vehicle + morphine treatment on day 8 ( $21.51 \pm 3.44$ vs. $12.76 \pm 2.78 \mathrm{sec} ; \mathrm{P}<0.001)$. Pretreatment with AM630 (AM630 + AM1241 + morphine group) produced a significant decrease in the effect of AM1241 on morphine analgesia in the treatment of cancer pain $(\mathrm{P}<0.001 ;$ Fig. 1).

Effect of coadministration of AM1241 and morphine on the development of morphine tolerance. The tumor-bearing rats treated with morphine for 8 days $(10 \mathrm{mg} / \mathrm{kg}$ twice daily) exhibited significantly decreased analgesic responses at 30,60 , 90 and 120 min compared with the vehicle + saline treatment group (all $\mathrm{P}<0.001$ ) when challenged with $5 \mathrm{mg} / \mathrm{kg}$ of morphine on day 9 (Fig. 2). The antinociceptive responses of $5 \mathrm{mg} / \mathrm{kg}$ morphine were significantly increased in the rats pretreated with the non-analgetic dose of AM1241 (AM1241 + morphine group) compared with those of the morphine-tolerant rats (vehicle + morphine group) at 30, 60, 90 and $120 \mathrm{~min}$ (all $\mathrm{P}<0.001$; Fig. 2). The selective CB2 antagonist AM630 completely reversed the effects of AM1241 on morphine tolerance (all $\mathrm{P}<0.001$; Fig. 2).

Effect of coadministration of AM1241 and morphine on the morphine-mediated TRPV1 protein expression level in the lumbar DRG. Immunohistochemistry revealed TRPV1 protein expression in DRG sensory neurons ipsilateral to the
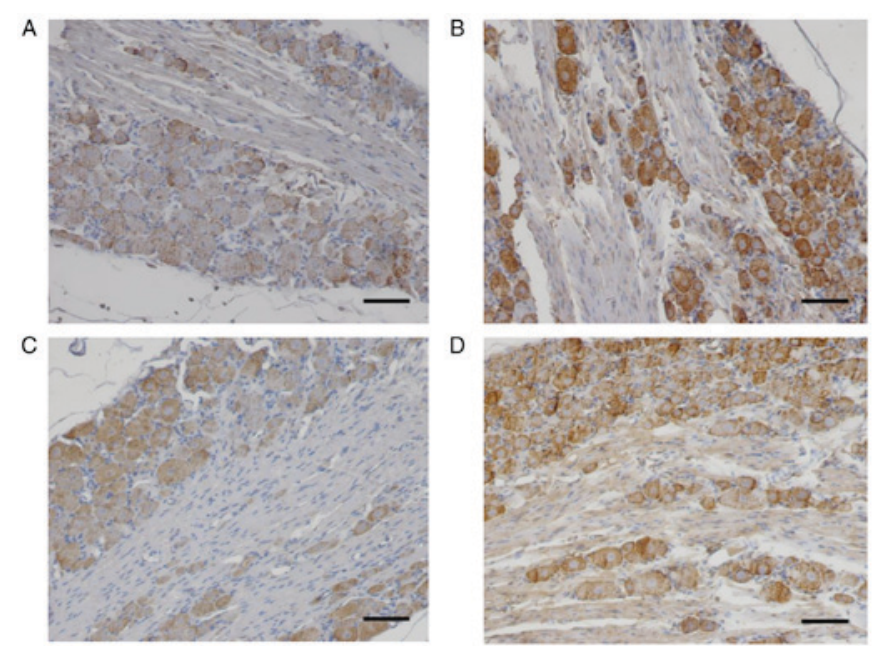

E

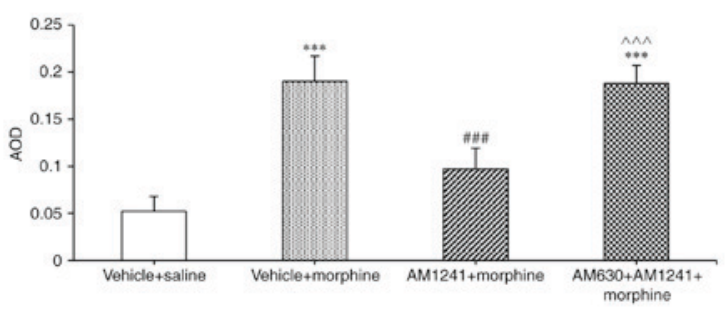

Figure 3. Immunohistochemical staining of TRPV1 in rat L4 DRG sections. (A) Vehicle + saline group $(n=4)$. (B) Vehicle + morphine group $(n=4)$. (C) AM1241 + morphine group (n=4). (D) AM630 + AM1241 + morphine group $(n=4)$. (E) AOD in slices of DRG. The values are presented as the mean \pm standard deviation. Scale bar, $100 \mu \mathrm{m} .{ }^{* * * *} \mathrm{P}<0.001$ vs. the

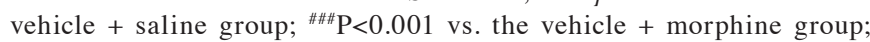
${ }^{\wedge \wedge} \mathrm{P}<0.001 \mathrm{AM} 1241+$ morphine group vs. the AM630 + AM1241 + morphine group. TRPV1, vanilloid receptor 1; DRG, dorsal root ganglia; AOD, average optical density.

site of tumor cell injection (Fig. 3). Chronic treatment with morphine for 8 days significantly increased TRPV1 protein 
A

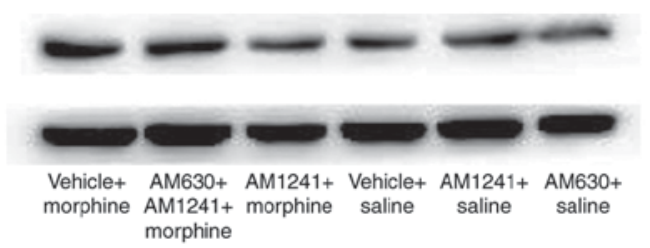

B

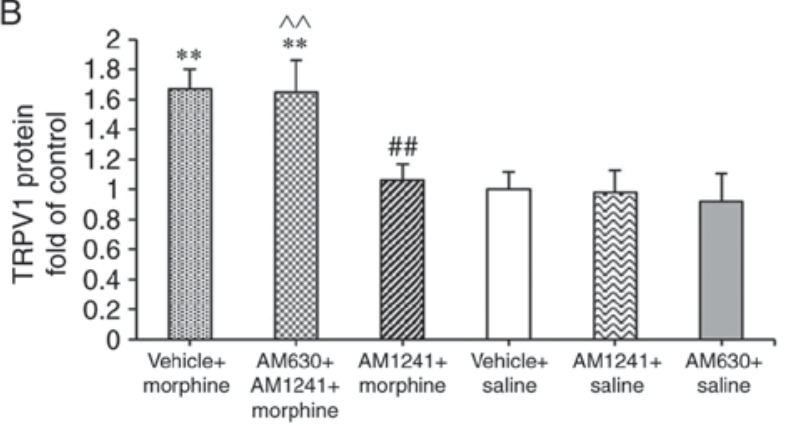

Figure 4. Western blotting of TRPV1. (A) Representative image of the western blotting of TRPV1 total protein expression in the L3/L4/L5 DRG at the expected molecular weight of $95 \mathrm{kDa}$ ( $\mathrm{n}=3$ /group). (B) Semi-quantitative densitometry measurements revealed an increase in TRPV1 protein expression in the DRG after 8 days of treatment with morphine, and pretreatment with AM1241 (AM1241 + morphine) reduced TRPV1 expression compared with the vehicle + morphine group. The data are expressed as the mean fold-change in protein expression relative to the control \pm standard deviation. Vehicle + saline group acted as the control. ${ }^{* *} \mathrm{P}<0.01$ vs. the vehicle + saline group; ${ }^{\# \#} \mathrm{P}<0.01$ vs. the vehicle + morphine group; ${ }^{\wedge} \mathrm{P}<0.01$ $\mathrm{AM} 1241$ + morphine group vs. the AM630 + AM1241 + morphine group. TRPV1, vanilloid receptor 1; DRG, dorsal root ganglia.

expression predominantly in sensory neurons compared with vehicle + saline group $(\mathrm{P}<0.001$; Fig. $3 \mathrm{~A}, \mathrm{~B}$ and $\mathrm{E})$. Pretreatment with AM1241 (AM1241 + morphine group) significantly reduced morphine-induced TRPV1 protein expression in DRG sensory neurons $(\mathrm{P}<0.001$; Fig. $3 \mathrm{C}$ and $\mathrm{E})$. The average optical density in the DRG sections significantly decreased in AM1241 + morphine group compared with vehicle + morphine group $(\mathrm{P}<0.001 ;$ Fig. 3E). AM630 reversed the effect of AM1241 on morphine-induced TRPV1 expression $(\mathrm{P}<0.001$; Fig. 3D and $\mathrm{E})$.

Western blotting was further performed to assess the total protein levels of TRPV1 in the DRG. The total protein levels of TRPV1 in the DRG increased significantly after 8 days of treatment with morphine $(\mathrm{P}=0.003)$, AM1241-pretreated group significantly decreased the TRPV1 expression compared with treatment with morphine alone (AM1241 + morphine group vs. vehicle + morphine group; $\mathrm{P}=0.007 ;$ Fig. 4).

Effect of coadministration of AM1241 and morphine on the morphine-mediated TRPVI mRNA expression level in the lumbar DRG. RT-qPCR revealed no significant change in the TRPV1 mRNA levels in the DRG among six groups (all $\mathrm{P}=0.913$; Fig. 5).

\section{Discussion}

In the present study, it was observed that coadministration of a non-analgesic dose of the CB2 agonist AM1241 and morphine attenuated TRPV1 protein expression in the DRG of morphine-tolerant tumor-bearing rats. These results suggest

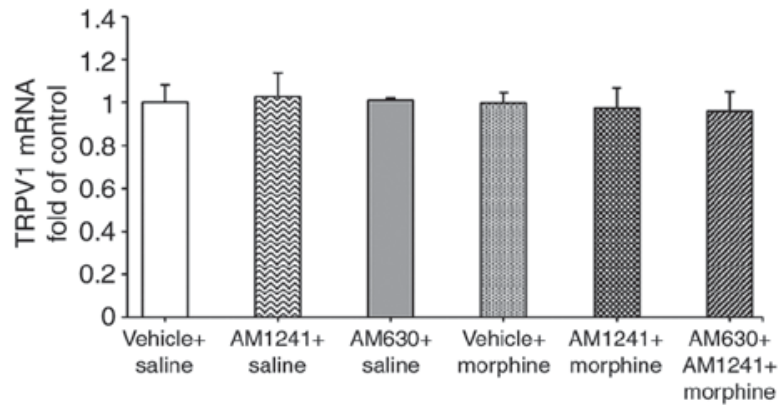

Figure 5. Reverse transcription-quantitative polymerase chain reaction analysis of TRPV1 mRNA expression in the L3/L4/L5 dorsal root ganglia ( $n=3 /$ group). There was no significant difference after 8 days of treatment with morphine (vehicle + morphine group) or pretreated with AM1241 (AM1241 + morphine group) compared with the vehicle + saline group. TRPV1, vanilloid 1 .

that downregulation of TRPV1 protein expression in the peripheral sensory neurons of the DRG may be one of the mechanisms by which coadministration of a CB2 agonist increased chronic morphine analgesia and attenuated tolerance in cancer pain.

In the present study, it was demonstrated that i.t. administration of a nonanalgetic dose of the CB2 agonist AM1241 potentiated morphine antinociception and alleviated morphine tolerance in a cancer pain rat model by measuring paw withdrawal latency to radiant heat stimulation. These results are consistent with the results of the authors' previous study using von Frey filament and hot plate test in Walker 256 tumor-bearing rats (18). It further confirmed that CB2 agonists may contribute to increased morphine antinociception and inhibit the development of tolerance associated with repeated treatment with morphine in cancer pain.

In primary sensory neurons, TRPV1 serves an important role in nociceptive conduction, including models of cancer, inflammatory and neuropathic pain $(20,24,27)$. It has been suggested that neuropathy or inflammation-induced hyperalgesia and morphine tolerance may share similar mechanisms (28-30). Following chronic treatment with morphine, the percentage of TRPV1-immunoreactive DRG neurons was increased (21), and activation of TRPV1 contributed to morphine tolerance in normal rats $(21,22)$. In the present study, the results revealed that chronic treatment with morphine resulted in increased expression levels of TPRV1 protein. These results implied that involvement of the TRPV1 in morphine tolerance in cancer pain. Previous studies reported that there is no increase in TRPV1 mRNA expression levels in the DRG in inflammation and normal rats with morphine tolerance $(21,31)$. In the present study, no increase in TRPV1 mRNA expression was detected in the lumbar DRG of morphine-tolerant rats in the rat model of cancer pain. The results suggested that chronic treatment with morphine enhances TRPV1 protein expression at the translational or post-translational level in DRG neurons (21).

Combination of morphine and TRPV1 antagonist potentiated the analgesic effects of morphine in a mouse model of bone cancer pain (32). I.t. and i.p. injection of a selective TRPV1 antagonist decreased TRPV1-immunoreactivity, and attenuated morphine tolerance and dependence in normal 
rats (21). A previous study demonstrated the colocalization of CB2 and TRPV1 in human DRG sensory neurons (12). The cannabinoids inhibited capsaicin responses in cultured human DRG neurons and in rat threonine ganglia $(12,23,33)$, and regulated TRPV1 in the animal model of inflammatory and neuropathic pain (24). However, little is known about the effect of CB2 agonist on TRPV1 expression on morphine tolerance in cancer pain. In the present study, it was observed that coadministration of a nonanalgetic dose of AM1241 and morphine significantly decreased TRPV1 immunostaining and total protein expression in the DRG following repeated treatment with morphine in the rat model of cancer pain, and the effect of the CB2 agonist AM1241 was abolished by the CB2 antagonist AM630. These results suggested a potential role for the CB2 agonist on TRPV1 expression in morphine tolerance in cancer pain.

CB2 and TRPV1 belong to the G-protein-coupled receptor family, Anand et al (12) demonstrated colocalization of CB2 and TRPV1 in human and rat DRG small/medium-diameter sensory neurons. A previous study reported that the increase in TRPV1 immunoreactivity contributes to morphine tolerance in a mitogen-activated protein kinase (MAPK)-dependent manner (21). Inhibition of MAPK phosphorylation reduced the increased TRPV1 protein expression that is normally associated with chronic treatment with morphine (21), and reduced morphine tolerance in normal rats and mice $(21,34)$. It has been demonstrated that CB2 agonists reduce the phosphorylated forms of MAPKs, including phosphorylated (p)-p38 and p-extracellular-regulated kinase $1 / 2$ in vitro and in a rat model of neuropathic pain $(35,36)$, which may subsequently regulate TRPV1 downstream of the DRG neurons of rats exhibiting morphine tolerance. In addition, CB2 agonists have been demonstrated to indirectly mediate TRPV1 phosphorylation by inhibiting adenylyl cyclase and depleting cAMP in cells, including cultured human DRG neurons, the BV2 microglial cell line, CB2-transfected human embryonic kidney cells and Chinese hamster ovary cells $(12,36)$, which possibly participates in CB2 agonist-associated morphine tolerance.

The mechanism of opioid tolerance remains unclear. CB2 agonist-associated reduced morphine tolerance may involve multiple aspects. The authors previously reported that a nonanalgetic dose of CB2 agonist AM1241 reduced morphine tolerance by upregulating $\mu$-opioid receptor protein and mRNA expression in a rat model of cancer pain (18). In addition, the activation of glial cells by chronic morphine administration contributes to morphine tolerance $(2,37)$. It is possible that the CB2 agonist alleviates morphine tolerance by reducing glial activation (4).

There are certain limitations to the present study. Specific TRPV1 inhibitors were not used to further elucidate the association between CB2 agonists and TRPV1 in morphine tolerance in the treatment of cancer pain; therefore, no direct proof of this result can be drawn from the data. The pathway between CB2 and TRPV1 was not investigated. Therefore, the molecular and cellular mechanisms involved in CB2 agonists-modulated TRPV1 expression in regards to analgesia and tolerance of morphine in cancer pain remain to be determined. Further studies are required to address these limitations.
In conclusion, the data indicated that coadministration of a nonanalgetic dose of a CB2 agonist and morphine reduced morphine tolerance in a rat model of cancer pain, and the effect of the CB2 agonist on morphine tolerance may involve the suppression of TRPV1 protein expression. The results provide further evidence that CB2 agonists may assist in the reduction of morphine tolerance in cancer pain, and also provide a novel strategy to strengthen morphine analgesia and improved clinical treatment of cancer pain.

\section{Acknowledgements}

The present study was supported by the Translational Medicine Special Foundation of China Russia Medical Research Center (grant nos. 201519 and CR1418), Natural Science Foundation of China (grant no. 81571885) and Haiyan Research Fund of Cancer Hospital of Harbin Medical University (grant no. JJQN2014-09).

\section{References}

1. Mercadante S: The use of opioids for treatment of cancer pain. Expert Opin Pharmacother 16: 389-394, 2015.

2. Watkins LR, Hutchinson MR, Johnston IN and Maier SF: Glia: Novel counter-regulators of opioid analgesia. Trends Neurosci 28: 661-669, 2005.

3. Nagi K and Piñeyro G: Regulation of opioid receptor signalling: Implications for the development of analgesic tolerance. Mol Brain 4: 25, 2011.

4. Tumati S, Largent-Milnes TM, Keresztes A, Ren J, Roeske WR, Vanderah TW and Varga EV: Repeated morphine treatment-mediated hyperalgesia, allodynia and spinal glial activation are blocked by co-administration of a selective cannabinoid receptor type-2 agonist. J Neuroimmunol 244: 23-31, 2012.

5. Ledent C, Valverde O, Cossu G, Petitet F, Aubert JF, Beslot F, Böhme GA, Imperato A, Pedrazzini T, Roques BP, et al: Unresponsiveness to cannabinoids and reduced addictive effects of opiates in CB1 receptor knockout mice. Science 283: 401-404, 1999.

6. De Vry J, Jentzsch KR, Kuhl E and Eckel G: Behavioral effects of cannabinoids show differential sensitivity to cannabinoid receptor blockade and tolerance development. Behav Pharmacol 15: 1-12, 2004.

7. Pertwee RG: Cannabinoid receptors and pain. Prog Neurobiol 63: 569-611, 2001.

8. Beltramo M, Bernardini N, Bertorelli R, Campanella M, Nicolussi E, Fredduzzi S and Reggiani A: CB2 receptor-mediated antihyperalgesia: Possible direct involvement of neural mechanisms. Eur J Neurosci 23: 1530-1538, 2006.

9. Romero-Sandoval A, Nutile-McMenemy N and DeLeo JA: Spinal microglial and perivascular cell cannabinoid receptor type 2 activation reduces behavioral hypersensitivity without tolerance after peripheral nerve injury. Anesthesiology 108: 722-734, 2008

10. Onaivi ES, Ishiguro H, Gong JP, Patel S, Perchuk A, Meozzi PA, Myers L, Mora Z, Tagliaferro P, Gardner E, et al: Discovery of the presence and functional expression of cannabinoid CB2 receptors in brain. Ann N Y Acad Sci 1074: 514-536, 2006.

11. Onaivi ES, Ishiguro H, Gong JP, Patel S, Meozzi PA, Myers L, Perchuk A, Mora Z, Tagliaferro PA, Gardner E, et al: Functional expression of brain neuronal $\mathrm{CB} 2$ cannabinoid receptors are involved in the effects of drugs of abuse and in depression. Ann N Acad Sci 1139: 434-449, 2008.

12. Anand U, Otto WR, Sanchez-Herrera D, Facer P, Yiangou Y, Korchev Y, Birch R, Benham C, Bountra C, Chessell IP and Anand P: Cannabinoid receptor CB2 localisation and agonist-mediated inhibition of capsaicin responses in human sensory neurons. Pain 138: 667-680, 2008.

13. Lim G, Wang S and Mao J: Central glucocorticoid receptors modulate the expression of spinal cannabinoid receptors induced by chronic morphine exposure. Brain Res 1059: 20-27, 2005.

14. Desroches J, Bouchard JF, Gendron L and Beaulieu P: Involvement of cannabinoid receptors in peripheral and spinal morphine analgesia. Neuroscience 261: 23-42, 2014. 
15. Cichewicz DL and Welch SP: Modulation of oral morphine antinociceptive tolerance and naloxone-precipitated withdrawal signs by oral Delta 9-tetrahydrocannabinol. J Pharmacol Exp Ther 305: 812-817, 2003.

16. Cichewicz DL: Synergistic interactions between cannabinoid and opioid analgesics. Life Sci 74: 1317-1324, 2004.

17. Bushlin I, Rozenfeld R and Devi LA: Cannabinoid-opioid interactions during neuropathic pain and analgesia. Curr Opin Pharmacol 10: 80-86, 2010.

18. Zhang M, Wang K, Ma M, Tian S, Wei N and Wang G: Low-dose cannabinoid type 2 receptor agonist attenuates tolerance to repeated morphine administration via regulating $\mu$-Opioid Receptor expression in Walker 256 tumor-bearing rats. Anesth Analg 122: 1031-1037, 2016.

19. Ma W and Quirion R: Inflammatory mediators modulating the transient receptor potential vanilloid 1 receptor: Therapeutic targets to treat inflammatory and neuropathic pain. Expert Opin Ther Targets 11: 307-320, 2007.

20. Zhang Z, Wang C, Gu G, Li H, Zhao H, Wang K, Han F and Wang G: The effects of electroacupuncture at the ST36 (Zusanli) acupoint on cancer pain and transient receptor potential vanilloid subfamily 1 expression in Walker 256 tumor-bearing rats. Anesth Analg 114: 879-885, 2012.

21. Chen Y, Geis C and Sommer C: Activation of TRPV1 contributes to morphine tolerance: Involvement of the mitogen-activated protein kinase signaling pathway. J Neurosci 28: 5836-5845, 2008.

22. Nguyen TL, Nam YS, Lee SY, Kim HC and Jang CG: Effects of capsazepine, a transient receptor potential vanilloid type 1 antagonist, on morphine-induced antinociception, tolerance, and dependence in mice. Br J Anaesth 105: 668-674, 2010.

23. Patwardhan AM, Jeske NA, Price TJ, Gamper N, Akopian AN and Hargreaves KM: The cannabinoid WIN 55,212-2 inhibits transient receptor potential vanilloid 1 (TRPV1) and evokes peripheral antihyperalgesia via calcineurin. Proc Natl Acad Sci USA 103: 11393-11398, 2006.

24. Devesa I and Ferrer-Montiel A: Neurotrophins, endocannabinoids and thermo-transient receptor potential: A threesome in pain signalling. Eur J Neurosci 39: 353-362, 2014.

25. Brigatte P, Sampaio SC, Gutierrez VP, Guerra JL, Sinhorini IL, Curi R and Cury Y: Walker 256 tumor-bearing rats as a model to study cancer pain. J Pain 8: 412-421, 2007.

26. Wang ZB, Gan Q, Rupert RL, Zeng YM and Song XJ: Thiamine, pyridoxine, cyanocobalamin and their combination inhibit thermal, but not mechanical hyperalgesia in rats with primary sensory neuron injury. Pain 114: 266-277, 2005.
27. Szallasi A, Cortright DN, Blum CA and Eid SR: The vanilloid receptor TRPV1: 10 years from channel cloning to antagonist proof-of-concept. Nat Rev Drug Discov 6: 357-372, 2007.

28. Ossipov MH, Lai J, King T, Vanderah TW and Porreca F: Underlying mechanisms of pronociceptive consequences of prolonged morphine exposure. Biopolymers 80: 319-324, 2005.

29. Mao J, Price DD and Mayer DJ: Mechanisms of hyperalgesia and opiate tolerance: A current view of their possible interactions. Pain 62: 259-2274, 1995.

30. Mayer DJ, Mao J, Holt J and Price DD: Cellular mechanisms of neuropathic pain, morphine tolerance, and their interactions. Proc Natl Acad Sci USA 96: 7731-7736, 1999.

31. Endres-Becker J, Heppenstall PA, Mousa SA,Labuz D, Oksche A, Schäfer M, Stein C and Zöllner C: Mu-opioid receptor activation modulates transient receptor potential vanilloid 1 (TRPV1) currents in sensory neurons in a model of inflammatory pain. Mol Pharmacol 71: 12-18, 2007.

32. Niiyama Y, Kawamata T, Yamamoto J, Furuse S and Namiki A: SB366791, a TRPV1 antagonist, potentiates analgesic effects of systemic morphine in a murine model of bone cancer pain. $\mathrm{Br} J$ Anaesth 102: 251-258, 2009.

33. Jeske NA, Patwardhan AM, Gamper N, Price TJ, Akopian AN and Hargreaves KM: Cannabinoid WIN 55,212-2 regulates TRPV1 phosphorylation in sensory neurons. J Biol Chem 281: 32879-32890, 2006.

34. Chen Y and Sommer C: The role of mitogen-activated protein kinase (MAPK) in morphine tolerance and dependence. Mol Neurobiol 40: 101-107, 2009.

35. Landry RP, Martinez E, DeLeo JA and Romero-Sandoval EA: Spinal cannabinoid receptor type 2 agonist reduces mechanical allodynia and induces mitogen-activated protein kinase phosphatases in a rat model of neuropathic pain. J Pain 13: 836-848, 2012.

36. Dhopeshwarkar A and Mackie K: CB2 Cannabinoid receptors as a therapeutic target-what does the future hold? Mol Pharmacol 86: 430-437, 2014.

37. Cui Y, Chen Y, Zhi JL, Guo RX, Feng JQ and Chen PX: Activation of p38 mitogen-activated protein kinase in spinal microglia mediates morphine antinociceptive tolerance. Brain Res 1069: 235-243, 2006. 